\title{
Modafinil Attenuates Disruptions in Cognitive Performance During Simulated Night-Shift Work
}

\author{
Carl L Hart*,, ,2, Margaret Haney', Suzanne K Vosburg', Sandra D Comer', Erik Gunderson' and \\ Richard W Foltin' \\ 'Department of Psychiatry, Division on Substance Abuse, New York State Psychiatric Institute, College of Physicians and Surgeons of Columbia \\ University, New York, NY, USA; ${ }^{2}$ Department of Psychology, Columbia University, New York, NY, USA
}

\begin{abstract}
Common complaints among shift workers are sleep disruptions and increased sleepiness while working, which may contribute to shift workers being more susceptible to diminished performance and work-related accidents. The purpose of this double-blind, withinparticipant study was to examine the effects of the alerting agent modafinil on cognitive/psychomotor performance, mood, and measures of sleep during simulated shift work. In all, I I participants completed this 23-day residential laboratory study. They received a single oral modafinil dose $(0,200,400 \mathrm{mg})$ I h after waking for three consecutive days under two shift conditions: day shift and night shift. Shifts alternated three times during the study, and shift conditions were separated by an 'off' day. When participants received placebo, cognitive performance and subjective ratings of mood were disrupted during the night shift, relative to the day shift. Objective and subjective measures of sleep were also disrupted, but to a lesser extent. Modafinil reversed disruptions in cognitive performance and mood during the night shift. While modafinil produced few effects on sleep measures during the night shift, the largest dose produced several sleep alterations during the day shift. These data demonstrate that abrupt shift changes produced cognitive performance impairments and mood disruptions during night shift work. Therapeutic doses of modafinil attenuated night-shift-associated disruptions, but the larger dose produced some sleep impairments when administered during day-shift work.

Neuropsychopharmacology (2006) 31, I526-1536. doi:I0. I038/sj.npp. I 300991; published online I4 December 2005
\end{abstract}

Keywords: Provigi ${ }^{\mathbb{R}}$; humans; sleep; performance; stimulant; mood

\section{INTRODUCTION}

Individuals who are required to work irregular or rotating shifts (eg, healthcare workers and military personnel) frequently adjust their sleep-wake cycles and report sleep disruptions and increased sleepiness while working (US Congress and Office of Technology Assessment, 1991). These conditions may contribute to diminished performance, disrupted mood, and work-related accidents. Indeed, a recent report indicated that rotating shift workers, relative to fixed daytime schedule workers, were not only more likely to report feeling sleepy at work, but were also more likely to have work-related accidents, possibly due to impaired performance (Ohayon et al, 2002). Rotating shift workers, particularly night-shift workers, may experience sleep disruptions because they attempt to sleep at a time

*Correspondence: Dr CL Hart, Department of Psychiatry, New York State Psychiatric Institute, Columbia University, 105। Riverside Drive, Unit 120, New York, NY 10032, USA, Tel: + I 212543 5884, Fax: + | 212543 599|, E-mail: clh42@columbia.edu

Received 27 April 2005; revised 21 October 2005; accepted 24 October 2005

Online publication: 28 October 2005 at http://www.acnp.org/citations/ Npp I02805050272/default.pdf that conflicts with their internal circadian clock (Eastman and Martin, 1999). Additionally, these workers are often required to perform at the circadian nadir of alertness. Together, these conditions may render individuals working rotating shifts less vigilant and more susceptible to performance decrements.

One strategy used to offset shift change-related disruptions is the administration of psychostimulants. Caffeine during a night shift, for example, has been demonstrated to improve performance (Walsh et al, 1990), increase ratings of alertness, and decrease physiological measures of sleep during work hours without altering daytime sleep (Muehlbach and Walsh, 1995). In addition, we reported that methamphetamine attenuated night-shift-related performance impairments and even improved performance on some measures that were not disrupted as a function of the night-shift condition (Hart et al, 2003a). Although these data clearly show that methamphetamine tempered nightshift-related behavioral disruptions, there is concern that night-shift workers may be more susceptible to methamphetamine abuse because of its performance and moodenhancing properties. Methamphetamine has been shown to have abuse potential in the laboratory (Hart et al, 2001b), but the abuse potential of methamphetamine in night-shift workers remains unknown. 
An alternative medication to methamphetamine is the alerting agent modafinil, which is chemically and pharmacologically distinct from other psychostimulants. Although the exact neural mechanisms that mediate modafinil-related effects remain unknown, increasing evidence indicate that modafinil-related therapeutic effects are dependent upon augmentation of catecholaminergic activity. Results from microdialysis studies show that modafinil increases extracellular dopamine concentrations in the striatum and prefrontal cortex (Wisor et al, 2001; de Saint Hilaire et al, 2001); this effect appears to be mediated via the dopamine transporter, as it is the only documented central nervous system (CNS) binding site for modafinil (Mignot et al, 1994). In addition, a substantial amount of data indicates that an intact $\alpha_{1}$-adrenergic receptor system is required for the wake-promoting effects of modafinil. For example, several researchers have reported that $\alpha_{1}$ antagonists block the effects of modafinil (eg, Duteil et al, 1990; Lin et al, 1992), although modafinil-related wake-promoting effects are unaltered by dopamine antagonists (Lin et al, 1992). Note also that noradrenergic projections from the locus coeruleus to the forebrain do not appear to be necessary for the wake-promoting actions of modafinil because chemical destruction of terminals along this major noradrenergic pathway does not produce alterations of modafinil's actions (Wisor and Eriksson, 2005). These findings suggest that modafinil-related neurobiological mechanism of action is complex and may not conform to simplistic views of neurotransmission. A growing body of evidence indicates that dopamine may act as an endogenous ligand at adrenergic receptors in several CNS regions (eg, Malenka and Nicoll, 1986; Crochet and Sakai, 2003). Thus, recently modafinil has been hypothesized to exert its therapeutic effects by increasing dopaminergic activity (ie, blockade of the dopamine transporter), which, in turn, stimulate adrenergic receptors (Wisor and Eriksson, 2005). Although modafinil increases catecholaminergic activity, it appears to have low abuse potential (Jasinski, 2000; Rush et al, 2002). It is important to note that modafinil also increases CNS glutamatergic activity (Ferraro et al, 1998), which may further contribute to its therapeutic actions.

Modafinil has been shown to improve performance impaired by fatigue (Pigeau et al, 1995) without producing disruptive effects on subsequent sleep (Buguet et al, 1995), and was recently approved in the US (Schedule IV controlled substance) for treating shift work sleep disorder (Schwartz, 2005). Moreover, Walsh et al (2004) investigated the effects of a single active modafinil dose $(0,200 \mathrm{mg})$ on the behavior of volunteers subjected to simulated night-shift work, and reported that the drug lessened some cognitive/ psychomotor deficits and decreased sleepiness during work periods. Modafinil did not improve performance on all cognitive/psychomotor tasks, however. Given that $200 \mathrm{mg}$ is a relatively low modafinil dose, we feel that further studies examining a broader dosing range are warranted. One question of interest was to determine if modafinil-related effects on night-shift disruptions are dose-dependent. In general, data from our study of methamphetamine indicated that attenuation of night-shift impairments was dose-related (Hart et al, 2003a). Another question of interest was to determine if modafinil attenuated shift change-related disruptions when research participants were subjected to abrupt shift changes. The study design employed by Walsh et al (2004) was not suited to address this issue because participants in that study did not undergo abrupt shift changes. In contrast, we have developed a laboratory model of shift work in which participants work several days on the 0830-1730-h shift, and several days on the 0030-0930-h shift, and switch between shifts several times during each study (Hart et al, 2003a, b, 2005). This model facilitates the examination of abrupt work shift change-related alterations in human behavior and the interactive effects of drugs on these alterations.

\section{METHODS}

\section{Participants}

In all, 11 healthy research volunteers (mean age $25.2 \pm 5.4$ $( \pm S D))$ completed this 23 -day residential study: five were females (four Black, one White) and six were males (one Asian, three Black, one Hispanic, one White). Participants' formal education ranged from 11 to 16 years (mean $=14.3$ ). They were solicited via word-of-mouth referral and newspaper advertisement in New York City. All participants reported current caffeine use (3-21 cups per week) and eight reported current alcohol use (1-7 drinks per week). Five participants reported marijuana use within the past year (use ranged from once per month to twice per year), and five smoked 2-20 tobacco cigarettes per day. Two participants reported using cocaine at least once. Urine toxicology analyses during the screening process confirmed the absence of illicit drug use. All participants reported previous experience working irregular shift schedules, passed comprehensive medical and psychological evaluations, and were within normal weight ranges according to the 1983 Metropolitan Life Insurance Company height/ weight table (body mass index: $24.3 \pm 3.2 \mathrm{~kg} / \mathrm{m}^{2}$ ).

Volunteers were informed that the purpose of the study was to evaluate the effects of an FDA-approved medication on the cognitive performance and mood of shift workers; all signed a consent form approved by the New York State Psychiatric Institute's Institutional Review Board. At the end of the study, volunteers were fully informed about experimental and drug conditions and were paid for their participation. They were compensated at a rate of $\$ 70$ per day.

\section{Laboratory}

Three groups of 3-4 individuals stayed in a residential laboratory in the New York State Psychiatric Institute (Foltin et al, 1996; Hart et al, 2003a). The laboratory has a common social area, where participants were free to engage in recreational activities. Each participant had a bedroom with a bed, desk, Macintosh computer system, microwave, toaster, refrigerator, and food preparation space. For the purpose of continuous observation of behavior, cameras and microphones are located throughout the common social area and in bedrooms, but not in bathrooms, showers, or private dressing areas. Communication between the staff and participants was kept to a minimum and was accomplished primarily via computers in each participant's bedroom. 


\section{Pre-Study Training}

Prior to study commencement, participants completed two training sessions (3-4h per session) on computerized tasks that would be used in the study and were familiarized with the laboratory and study procedures. On a separate day, they received a modafinil capsule $(400 \mathrm{mg})$ to provide them with experience with the study drug and to monitor any potential unusual reactions to the medication. No untoward events were observed.

\section{Design}

This 23-day residential study consisted of six 3-day blocks of sessions, during which participants completed computerized task batteries on two different shifts. During the day shift, they performed computer tasks from 0830 to $1730 \mathrm{~h}$ and went to bed at $2400 \mathrm{~h}$; during the night shift, they performed computer tasks from 0030 to $0930 \mathrm{~h}$ and went to bed at $1600 \mathrm{~h}$. Shifts alternated three times during the study and shift conditions were separated by an 'off day, during which participants were not on a work schedule but were required to go to bed $8 \mathrm{~h}$ prior to the next shift as they had done during other days. Two groups of participants started on the night shift and the other started on the day shift. Placebo or modafinil $(200,400 \mathrm{mg})$ was administered once per day, $1 \mathrm{~h}$ after waking ( 0915 on the day shift and 0115 on the night shift). Days 8 and 16 were 'drug washout' days, during which participants received placebo modafinil before being switched to another drug condition. In order to minimize potential confounding effects, presentation of modafinil doses were systematically varied between and within groups of participants. All participants experienced six dose/shift combinations: placebo + day and night shift, low dose + day and night shift, and high dose + day and night shift.

\section{Procedure}

Volunteers moved into the laboratory on the day before study commencement so that they could receive further training on tasks and experimental procedures. The first experimental day began at $0015 \mathrm{~h}$ (ie, the night shift) for seven participants and at $0815 \mathrm{~h}$ (ie, the day shift) for four participants the following morning. Participants first completed baseline cognitive/psychomotor tasks, a 44-item subjective-effects visual analog questionnaire, and a visual analog sleep questionnaire (see below). Then, they were weighed (but were not informed of their weight) and given time to eat breakfast. Following breakfast, eight $30-\mathrm{min}$ computerized task batteries, composed of the subjectiveeffects questionnaire and psychomotor tasks, were completed daily. Participants were given a 15-min break between each task battery. From 1000 (0200) to 1245 (0445) h, participants completed four task batteries and, after a 1.5-h lunch break period, they completed four additional task batteries from 1415 (0615) to $1700(0900) \mathrm{h}$. Beginning at $1700(0900) \mathrm{h}$, participants had access to activities available in the social area. Two films were shown daily, beginning at $1800(1000)$ and $2100(1300)$ h. Lights were turned out at $2400(1600) \mathrm{h}$ for an 8.25 -h sleep period.

\section{Subjective-Effects and Cognitive/Psychomotor Battery}

The computerized visual analog questionnaire consisted of a series of 100-mm lines labeled 'not at all' at one end and 'extremely' at the other end. The lines were labeled with 'I feel...,' 'Alert,' 'Angry,' 'Anxious,' 'a Bad Drug Effect,' 'Clumsy,' 'Confused,' 'Content,' 'Depressed,' 'Dizzy,' 'Energetic,' 'Forgetful,' 'Friendly,' 'a Good Drug Effect,' 'High,' 'Hungry,' 'Irritable,' 'Jittery,' 'Mellow,' 'Miserable,' 'Nauseous,' 'On Edge,' 'Restless,' 'Sedated,' 'Self-confident,' 'Sleepy,' 'Social,' 'Stimulated,' 'Talkative,' 'Tired,' 'Unmotivated,' 'Withdrawn,' 'I am Sweating,' 'I'm having Difficulty Concentrating,' 'I have Chills,' 'I have a Headache,' 'I have Muscle Pain,' 'I have Stomach Pain,' 'I have an Upset Stomach,' 'I want a Cigarette,' 'I want Marijuana,' 'My Heart is Pounding or Beating Faster than Usual,' 'My Vision is Blurred,' and 'Noises or Sounds Seem Louder than Usual.' Each day, a drug-effect questionnaire (DEQ) was also completed $90 \mathrm{~min}$ after modafinil administration. Participants were required to rate 'Good Effects' and 'Bad Effects' from the drug on a five-point scale: $0=$ 'not at all' and $4=$ 'very much.' They were also asked to rate how 'Strong' the drug effect was and their desire to 'take the drug again.' Lastly, participants were ask to rate how much they liked the drug effect on a 9-point scale: 4 indicated 'disliked very much,' 0 indicated 'feel neutral, or feel no drug effect,' and 4 indicated 'liked very much.'

The computerized cognitive/psychomotor battery consisted of five tasks: (1) the Digit Recall Task was designed to assess changes in immediate and delayed recall. An eightdigit number was displayed on the computer screen for $3 \mathrm{~s}$, and participants were instructed to enter the number correctly while it was on the screen and again after it had disappeared from the screen (Hart et al, 2001a). They were also informed that they would be asked to reproduce and recognize the number near the end of the battery; (2) the digit-symbol substitution task (DSST) was designed to assess changes in visuospatial processing. This task consisted of nine random three-row $\times$ three-column squares (one square blackened/row) displayed across the top of the computer screen (McLeod et al, 1982). Each array was associated with a number (1-9). A randomly generated number appeared at the bottom of the screen, indicating which of the arrays should be reproduced on the nine-key keypad attached to the computer. Participants were instructed to reproduce as many patterns as possible by entering the patterns associated with the randomly generated numbers; (3) the divided attention task (DAT) was designed to assess changes in vigilance and inhibitory control. This task consisted of concurrent pursuit-tracking and vigilance components (Miller et al, 1988). Participants were required to track a moving circle on the computer screen using the mouse and also had to signal (by clicking the mouse) when a small black square appeared at any of the four corners of the screen. Accurate tracking of the moving stimulus increased its speed proportionately; (4) the rapid information task (RIT) was designed to assess changes in sustained concentration and inhibitory control. During this task, a series of digits was displayed rapidly on the computer screen (100 digits/min), and participants were instructed to press a key as quickly as possible after three consecutive odd or even digits had appeared (Wesnes and Warburton, 
1983); and (5) the Repeated Acquisition Task was designed to assess changes in learning and memory. At the start of this task, four buttons were illuminated, and participants were instructed to learn a 10-response sequence of button presses (Kelly et al, 1993). A position counter incremented by one each time a correct button was pressed, and remained unchanged after an incorrect response. The points counter increased by 1 each time the 10-response sequence was correctly completed. The sequence remained the same throughout the task, but a new random sequence was generated when the task occurred again. Participants were instructed to earn as many points during the task as possible by pressing the buttons in the correct sequence.

\section{Sleep Monitoring}

The portable Nightcap sleep systems, which recorded objective measures of sleep, consist of a headband worn by participants while they slept (Ajilore et al, 1995; Cantero et al, 2002). A miniature electrode attached to the eyelid measured eye movement but did not disrupt sleep (Ajilore et al, 1995), and a body movement sensor in the headband detected and recorded movements. The system, which allows for measurement of total sleep time, sleep onset latency, rapid eye movement latency, non-rapid eye movement sleep time, and sleep efficiency (total sleep time as a percentage of time in bed), has been reported to correspond well to traditional polysomnography and subjective reports of sleep quality (Ajilore et al, 1995). In addition to wearing the portable sleep monitor, participants completed a visual analog sleep questionnaire each morning. This questionnaire consisted of a series of $100 \mathrm{~mm}$ lines labeled 'not at all' at one end and 'extremely' at the other end. The lines were labeled: 'I slept well last night,' 'I woke up early this morning,' 'I fell asleep easily last night,' 'I feel clear-headed this morning,' 'I woke up often last night,' 'I am satisfied with my sleep last night,' and a fill-in question in which participants were asked to estimate the number of hours they thought they slept the previous night (Haney et al, 2001).

\section{Drug}

Tablets of modafinil hydrochloride $(200 \mathrm{mg})$ (Provigil ${ }^{\circledR}$, Cephalon, Inc., West Chester, PA) were repackaged by the Pharmacy Department of the New York State Psychiatric Institute by placing tablets into a white $\# 00$ opaque capsule and adding lactose filler. Placebo consisted of white \#00 opaque capsules containing only lactose. All capsules were administered double-blind.

\section{Data Analysis}

Data from off and drug washout days (days 4, 8, 12, 16, 20) were not included in the analyses. In addition, 2 days of data from one volunteer were not included in the analyses because the volunteer was unable to complete these days due to a personal issue. The area under the curve (AUC) for the subjective-effects visual analog questionnaire and cognitive/psychomotor tasks was determined using the trapezoidal method (Tallarida and Murray, 1981). Peak subjective-effect and cognitive/psychomotor data were analyzed similarly. For the sake of brevity, data for the AUC analyses are discussed primarily because significant drug effects were similar when performing both peak and AUC analyses.

Data were analyzed using three-factor repeated-measures analyses of variance (ANOVA): the first factor was modafinil dose (placebo, 200, $400 \mathrm{mg}$ ), the second factor was shift condition (day, night), and the third factor was day within condition $(1,2,3)$. For all analyses, ANOVAs provided the error terms needed to calculate planned comparisons that were designed to answer two questions: (1) are cognitive/psychomotor task performance and subjective-effects ratings disrupted during the night shift, and (2) does modafinil attenuate night shift-related disruptions? To evaluate night shift-related disruptions, each day of placebo was compared to the corresponding night of placebo (eg, the first day of placebo during the day shift $v s$ the first night of placebo during the night shift). To evaluate the effects of modafinil on night shift-related disruptions, each night of each drug condition was compared to a corresponding night of another drug condition (eg, the first night of $200 \mathrm{mg}$ modafinil during the night shift $v s$ the first night of placebo during the night shift). Modafinil-related effects during day-shift work were evaluated similarly. Given the overall number of planned comparisons, only those $p$-values $<0.03$ were considered statistically significant in an effort to control for Type I error. Huynh-Feldt corrections were used when appropriate.

\section{RESULTS}

\section{Effects of Shift Condition}

Cognitive performance. The upper panels of Figure 1 illustrate how selected cognitive/psychomotor performance varied between the day and night shifts when participants received placebo. Planned comparisons revealed that the number of false alarms committed on the DAT significantly increased, while the number of correct digits recalled on the immediate recall task and total correct responses on the DSST significantly decreased during all three nights that participants worked on the night shift compared to the corresponding days on the day shift $(p<0.03)$. As shown in Tables 1-3, other performance measures (eg, hit latency on the DAT) also were altered significantly as a function of shift condition.

Subjective-effect ratings. The upper panels of Figure 2 show how selected subjective-effect ratings varied between the day and night shifts when participants received placebo. Ratings of 'Alert' were decreased during two nights that participants were on the night shift compared to the corresponding day shift days $(p<0.03)$. Conversely, ratings of 'Can't Concentrate' and 'Sleepy' were increased during two and three nights of the night shift (respectively), relative to the corresponding day shift days $(p<0.01)$. Tables 1-3 show that several other subjective-effect ratings (eg, 'Energetic') were altered significantly as a function of shift condition.

Sleep. Nightcap data from three participants were not available due to equipment malfunctions. The upper panels 
DAT: False Alarms
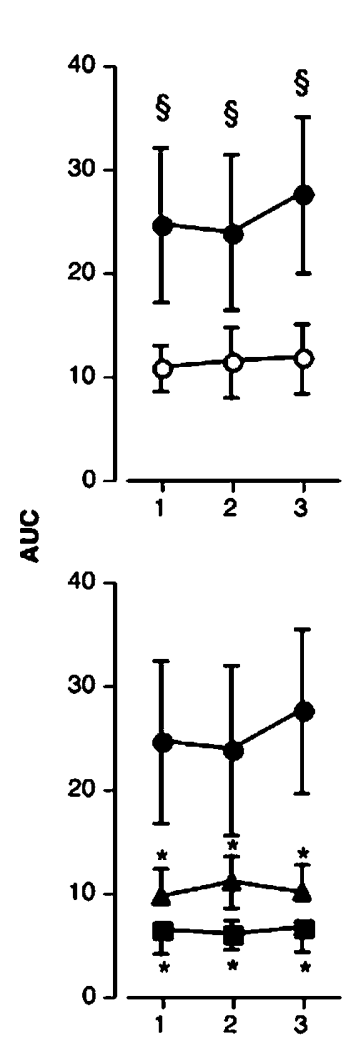

Digit Recall: Immediate

DSST: Total Correct
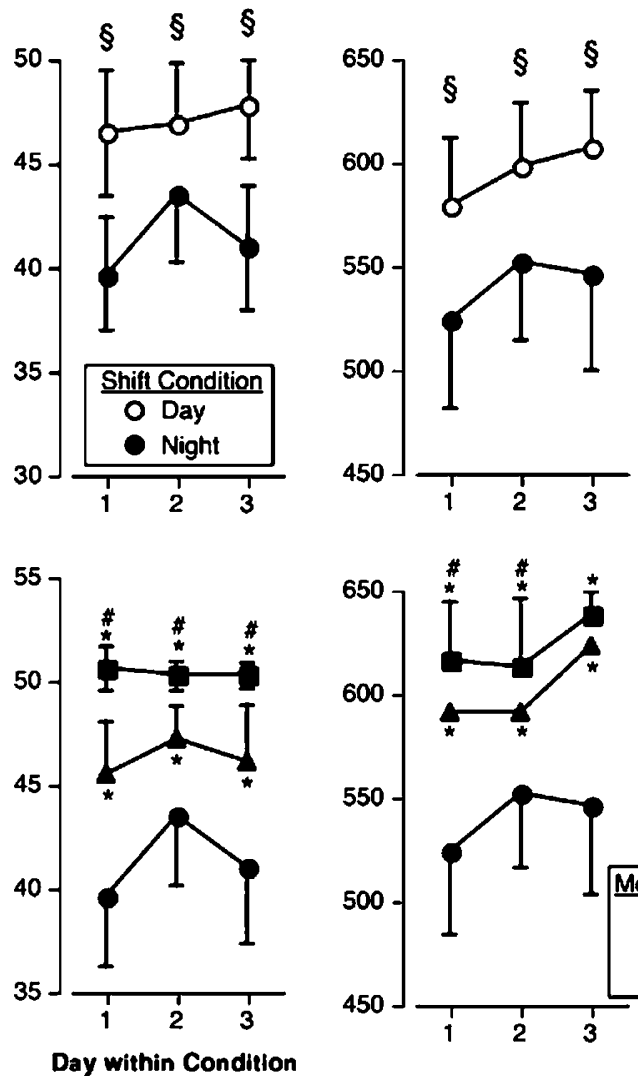
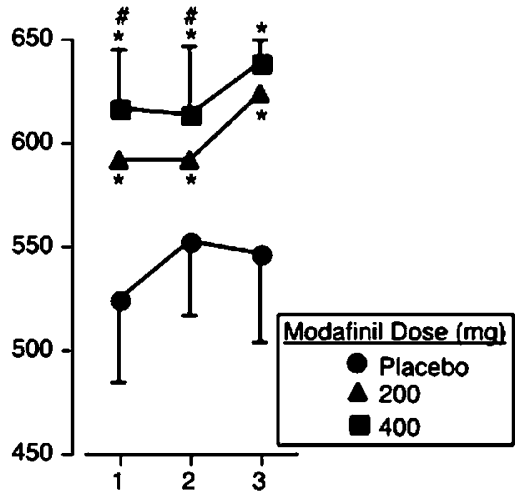

Figure I Upper panel: AUC values for false alarms (DAT), correct digits recalled, and total number correct (DSST) as a function of shift condition and day within condition. ${ }^{\$}$ Significant difference between the day and night shift conditions for that day following placebo administration ( $\left.p<0.03\right)$. Bottom panel: AUC values for false alarms (DAT), correct digits recalled, and total number correct (DSST) as a function of modafinil dose and day of the night-shift condition. *Significant difference between placebo and that dose of modafinil for that day $(p<0.03)$. "Significant difference between 200 and 400 mg modafinil for that day $(p<0.03)$. Error bars represent I SEM. Overlapping error bars were omitted for clarity.

of Figure 3 display how total sleep time and sleep onset latency, as measured by the Nightcap, varied between the day and night shifts when participants received placebo. Total sleep time was decreased across all three nights that participants worked on the night shift, but this effect was not statistically significant. Similarly, although sleep onset latency increased during night-shift work, this effect was statistically significant only on the third night $(p<0.01)$. Sleep efficiency was decreased when participants worked on the night shift compared with the day shift, but this effect only approached statistical significance on the first night $(p=0.039$, data not portrayed).

The bottom panels of Figure 3 indicate how selected ratings on the sleep questionnaire varied between the day and night shifts when participants received placebo. Upon waking before starting the first night-shift night, participants estimated that they had slept approximately 1.5 fewer hours the previous night, relative to when working on the day shift $(p<0.003)$. Additionally, participants reported having greater difficulties falling asleep the preceding evening, sleeping less well, and waking more frequently during the sleep period compared to the corresponding day-shift days ( $p<0.03$; some data not shown). These effects were statistically significant only on the first day of the night-shift condition.

\section{Effects of Modafinil}

Cognitive performance. The bottom panels of Figure 1 show how modafinil affected cognitive performance during the night-shift condition. (The night-shift data in the upper panels are the same data shown under the placebo condition in the bottom panels.) Active modafinil $(200,400 \mathrm{mg})$ decreased the number of false alarms on the DAT and increased the correct number of digits recalled on the immediate recall task, as well as the total number of correct responses on the DSST across all three nights $(p<0.01$ for all three nights). Tables 1-3 indicate that modafinil also improved cognitive performance on several other measures (eg, decreased the number of errors on the RA task) during night-shift work.

Relative to placebo, modafinil also improved cognitive/ psychomotor performance during the day-shift condition, although the effects were less dramatic than those observed under the night-shift condition (data not shown). On the DAT, both active doses increased tracking speed on the first day $(p<0.02)$. On the DSST, the $400-\mathrm{mg}$ dose increased the number of attempts and correct responses on days 1 and 2 $(p<0.001)$. On the RIT, both doses decreased total misses and increased the number of hits, while the $400-\mathrm{mg}$ dose decreased the number of false alarms $(p<0.008)$; these 
Table I Effects of Shift Condition and Modafinil on Psychomotor Performance and Subjective Effects on Day I

Conditions

\begin{tabular}{|c|c|c|c|c|c|c|c|}
\hline \multirow[b]{2}{*}{ Measure } & \multirow{2}{*}{$\begin{array}{c}\text { Pbo Day } \\
\text { Mean (SEM) }\end{array}$} & \multicolumn{2}{|c|}{ Pbo Night } & \multicolumn{2}{|c|}{$200 \mathrm{mg}$} & \multicolumn{2}{|c|}{$400 \mathrm{mg}$} \\
\hline & & Mean (SEM) & F-value & Mean (SEM) & $F$-value & Mean (SEM) & F-value \\
\hline DAT: hit latency & $7.23(0.68)$ & $8.80(0.9 \mid)$ & $12.58^{\S}$ & $7.20(0.84)$ & $13.13 *$ & $6.74(0.57)$ & $21.75^{*}$ \\
\hline DAT: speed & $54.36(2.92)$ & $49.36(3.29)$ & $9.34^{\S}$ & $51.64(3.31)$ & 1.93 & $58.18(2.40)$ & $29.04 *{ }^{\dagger}$ \\
\hline RA: total trials & $216.82(31.54)$ & I53.55 (27.00) & $18.03^{\S}$ & $|90.9|(26.88)$ & $6.29 *$ & $250.73(30.84)$ & $42.53^{* \dagger}$ \\
\hline RIT: false alarms & $297.64(60.52)$ & 335.18 (59.48) & 3.14 & $252.73(47.70)$ & $15.13^{*}$ & $264.18(47.23)$ & $11.22 *$ \\
\hline RIT: no. of hits & $1026.82(63.34)$ & 925.09 (78.95) & $14.16^{\S}$ & | | 42.27 (57.69) & $64.54 *$ & $1084.00(62.58)$ & $34.55^{*}$ \\
\hline RIT: no. of misses & $268.46(59.04)$ & $368.09(69.13)$ & $22.01^{\S}$ & | $81.82(53.58)$ & $77.25 *$ & $214.55(57.82)$ & $52.49 *$ \\
\hline Hunger & $55.9 \mid(23.82)$ & $19.09(9.46)$ & $10.80^{\S}$ & $36.91(22.46)$ & 2.53 & $29.18(12.93)$ & 0.81 \\
\hline Mellow & $177.18(70.11)$ & $|58.9|(62.13)$ & 0.74 & $218.18(60.89)$ & $7.82 *$ & $264.18(77.15)$ & $24.66 *$ \\
\hline Sedated & $4.09(3.42)$ & 101.55 (44.93) & $36.51^{\S}$ & $20.09(12.98)$ & $25.51 *$ & $45.55(34.86)$ & 12.06* \\
\hline Tired & | 44.64 (56.99) & 304.64 (59.29) & $15.36^{\S}$ & I $38.64(34.52)$ & | $6.53^{*}$ & $197.09(62.60)$ & $6.94 *$ \\
\hline Unmotivated & $56.29(31.49)$ & | $39.36(42.42)$ & $13.57^{\S}$ & $46.82(29.42)$ & |6.83* & | 34.82 (59.87) & 0.04 \\
\hline
\end{tabular}

Data are represented as AUC values and data from active modafinil conditions are presented for the night shift only.

$\mathrm{df}=\mathrm{I}, 40$.

$\mathrm{Pbo}=$ placebo; $\mathrm{DAT}=$ divided attention task; $\mathrm{RA}=$ repeated-acquisition task; $\mathrm{RIT}=$ rapid information task.

$\S_{p}<0.03$, significant difference between the placebo day and placebo night conditions.

$* p<0.03$, significant difference from the placebo night condition.

$p<0.03$, significant difference from the $200 \mathrm{mg}$ night condition.

effects were noted for all three days of day-shift work. On the repeated-acquisition task, $400 \mathrm{mg}$ modafinil decreased the number of errors made on days 1 and $3(p<0.03)$ and increased the number trials completed on day $1(p<0.003)$. Finally, the drug $(400 \mathrm{mg})$ increased the number of correct digits entered on the immediate recall task on days 1 and 2 $(p<0.03)$.

Subjective-effect ratings. The bottom panels of Figure 2 display how modafinil affected subjective-effect ratings during the night-shift condition. The $200-\mathrm{mg}$ dose increased ratings of 'Alert' on nights one and three, and the 400-mg dose increased ratings of 'Alert' on all three nights $(p<0.02)$. Both active doses decreased ratings of 'Can't concentrate' on at least two of the three nights $(p<0.02)$ and decreased ratings of 'Sleepy' on at least one of the three nights $(p<0.0007)$. While modafinil produced significant effects on several other subjective ratings (eg, 'Mellow', see Tables 1-3), measures from the DEQ were not altered significantly as a function of modafinil dose during nightshift work.

Consistent with its effects on cognitive/psychomotor performance, modafinil produced fewer statistically significant subjective effects during day-shift work. Both active doses increased ratings of 'Alert' on the first 2 days $(p<0.03)$. The 200-mg dose increased ratings of 'Heart pounding' on all 3 days and the $400-\mathrm{mg}$ dose increased these ratings on days 1 and $3(p<0.009)$. Finally, the $400-\mathrm{mg}$ modafinil dose increased ratings of 'Anxious' on the second and third days $(p<0.03)$.

Sleep. Modafinil produced only one significant effect on Nightcap sleep measures during night-shift work. Relative to placebo, the $400-\mathrm{mg}$ dose increased sleep onset latency on the third night: the mean sleep onset latency was $53 \mathrm{~min}$ under the placebo condition, compared to $146 \mathrm{~min}$ under the modafinil condition $(p<0.004)$. Data from the sleep questionnaire indicated that participants estimated that they had slept approximately 1.5 fewer hours and reported being less satisfied with their sleep following the 400-mg dose on the first night of night-shift work, compared to placebo and the $200-\mathrm{mg}$ dose $(p<0.009)$.

In contrast to its limited effects on sleep measures during the night-shift condition, modafinil produced several significant effects on sleep when participants worked on the day shift. Across the three days, total sleep time (as measured by the Nightcap) was decreased by an average of 67 min per night under the $400-\mathrm{mg}$ modafinil condition, relative to the placebo condition, although the effect of modafinil on total sleep time was statistically significant 
Table 2 Effects of Shift Condition and Modafinil on Psychomotor Performance and Subjective Effects on Day 2

Conditions

\begin{tabular}{|c|c|c|c|c|c|c|c|}
\hline \multirow[b]{2}{*}{ Measure } & \multirow{2}{*}{$\begin{array}{c}\text { Pbo Day } \\
\text { Mean (SEM) }\end{array}$} & \multicolumn{2}{|c|}{ Pbo Night } & \multicolumn{2}{|c|}{$200 \mathrm{mg}$} & \multicolumn{2}{|c|}{$400 \mathrm{mg}$} \\
\hline & & Mean (SEM) & F-value & Mean (SEM) & F-value & Mean (SEM) & F-value \\
\hline DAT: hit latency & $6.75(0.66)$ & $8.01(0.54)$ & $8.47^{\S}$ & $6.73(0.84)$ & 8.68* & $6.58(0.62)$ & $10.97 *$ \\
\hline DAT: speed & $56.00(1.73)$ & $51.09(3.48)$ & $9.00^{\S}$ & $54.27(3.04)$ & 3.78 & $60.36(1.97)$ & $32.11^{\dagger} \dagger$ \\
\hline RA: total trials & $238.18(24.35)$ & 175.09 (26.37) & $17.93^{\S}$ & $215.09(32.64)$ & $7.21 *$ & $241.27(23.19)$ & 19.73* \\
\hline RIT: false alarms & $289.36(61.58)$ & $396.64(68.01)$ & $25.61^{\S}$ & $222.9 \mid(39.82)$ & $67.16 *$ & $239.73(41.39)$ & $54.78 *$ \\
\hline RIT: no. of hits & 1042.09 (71.01) & 941.91 (68.48) & $13.73^{\S}$ & I I 69.46 (55.45) & $70.85 *$ & | I 07.00 (69.52) & $37.29 *$ \\
\hline RIT: no. of misses & $253.82(62.7 I)$ & $344.09(61.29)$ & $18.14^{\S}$ & I $64.27(52.76)$ & $71.99 *$ & 209.55 (62.05) & $40.31 *$ \\
\hline Irritable & $25.55(21.10)$ & $120.00(61.77)$ & $8.33^{\S}$ & $28.9 \mid(19.25)$ & 7.75 & $65.64(34.23)$ & 2.76 \\
\hline Mellow & $192.27(70.11)$ & $150.82(60.4 \mid)$ & 3.83 & $219.36(65.22)$ & $10.46 *$ & $242.64(72.71)$ & 18.76* \\
\hline Unmotivated & 48.27 (32.08) & | | 8.09 (44.36) & $9.58^{\S}$ & $59.27(32.21)$ & $6.80 *$ & | | 3.00 (52.28) & 0.05 \\
\hline
\end{tabular}

Data are represented as AUC values and data from active modafinil conditions are presented for the night shift only.

$\mathrm{df}=1,40$.

$\mathrm{Pbo}=$ placebo; $\mathrm{DAT}=$ divided attention task; $\mathrm{RA}=$ repeated-acquisition task; $\mathrm{RIT}=$ rapid information task.

$\S_{p}<0.03$, significant difference between the placebo day and placebo night conditions.

* $p<0.03$, significant difference from the placebo night condition.

${ }^{\dagger} p<0.03$, significant difference from the $200 \mathrm{mg}$ night condition.

only on day 3. On average, participants slept 331 min under the $400-\mathrm{mg}$ modafinil condition, compared with $440 \mathrm{~min}$ under the placebo condition $(p<0.003)$. The Nightcap measure of sleep efficiency showed a similar pattern. Under the 400-mg condition, sleep efficiency was significantly decreased following three consecutive days of drug treatment, relative to placebo treatment $(p<0.006)$ : on average, sleep efficiency was $65 \%$ the evening following modafinil $(400 \mathrm{mg})$ and $86 \%$ following placebo. In addition, sleep onset latency significantly increased during days 1 and 2 under the 400-mg condition, relative to the placebo and $200 \mathrm{mg}$ conditions $(p<0.02)$. On average, sleep onset latency was $116 \mathrm{~min}$ during the $400-\mathrm{mg}$ condition, compared with 46 and $30 \mathrm{~min}$ during the $200-\mathrm{mg}$ and placebo conditions. Similarly, results from the sleep questionnaire indicated that modafinil produced disruptions during dayshift work. Relative to placebo, participants reported having greater difficulties falling asleep on the first and second evenings following modafinil $(400 \mathrm{mg})$, and reported being less satisfied with their sleep and sleeping less well on the first evening following this dose of modafinil $(p<0.02)$.

\section{DISCUSSION}

The current data clearly show that cognitive/psychomotor performance, mood, and sleep measures were disrupted during the night shift compared to the day shift. These results are consistent with data from other investigations that assessed the effects of changing participants' work shift schedules under laboratory conditions (eg, Reid and Dawson, 2001; Sharkey et al, 2001; Hart et al, 2003a,b). Modafinil markedly improved nighttime performance and mood, while producing few deleterious effects on sleep. These findings replicate and extend results from another investigation that assessed the effects of one active modafinil dose $(200 \mathrm{mg})$ on night-shift performance and mood in research participants (Walsh et al, 2004). Note, however, that modafinil treatment during day-shift work was associated with a greater number of sleep disruptions.

When participants worked on the night shift and received placebo, they performed poorly on a wide range of tasks assessing several cognitive domains. For example, inhibitory control, as measured by two separate tasks (ie, DAT: false alarms and RIT: false alarms), was profoundly impaired. In light of the importance of inhibition as a basic unit of working memory and executive control processes (Dempster and Corkill, 1999; Zacks and Hasher, 1994), these data suggest that rotating shift workers may exhibit some executive cognitive functioning problems when working on the night shift, especially during the first few nights after a shift change. Other domains were also negatively affected during night-shift work, including diminished learning (eg, RA: total errors), impaired visuospatial processing (eg, DSST: total correct responses), and reduced sustained concentration or vigilance (eg, DAT: mean hit latency). The current data are consistent with reports that indicate that night-shift workers may be more 
Table 3 Effects of Shift Condition and Modafinil on Psychomotor Performance and Subjective Effects on Day 3

Conditions

\begin{tabular}{|c|c|c|c|c|c|c|c|}
\hline \multirow[b]{2}{*}{ Measure } & \multirow{2}{*}{$\begin{array}{c}\text { Pbo Day } \\
\text { Mean (SEM) }\end{array}$} & \multicolumn{2}{|c|}{ Pbo Night } & \multicolumn{2}{|c|}{$200 \mathrm{mg}$} & \multicolumn{2}{|c|}{$400 \mathrm{mg}$} \\
\hline & & Mean (SEM) & F-value & Mean (SEM) & F-value & Mean (SEM) & F-value \\
\hline DAT: speed & $57.55(2.5 \mathrm{I})$ & $51.73(3.89)$ & $12.64^{\S}$ & $57.64(3.31)$ & | 3.04 & $59.82(2.18)$ & 24.44* \\
\hline DSST: attempted & $633.46(33.49)$ & $574.46(49.46)$ & $62.16^{\S}$ & 641.91 (34.20) & $81.25 *$ & $656.9 \mid(35.7 \mid)$ & $\left.121.4\right|^{*}$ \\
\hline RIT: false alarms & 309.18 (57.73) & $379.36(74.55)$ & $10.96^{\S}$ & $199.46(32.37)$ & $72.02 *$ & $237.82(47.4 I)$ & $44.58 *$ \\
\hline RIT: no. of hits & I $036.46(79.79)$ & $961.36(87.74)$ & $7.72^{\S}$ & | |53.9| (52.74) & $50.73 *$ & I I 45.64 (72.35) & 46.46* \\
\hline RIT: no. of misses & $276.9 \mid(71.87)$ & $334.36(67.57)$ & $7.35^{\S}$ & $150.82(49.16)$ & $75.01 *$ & | 84.55 (61.27) & 49.98* \\
\hline \multicolumn{8}{|l|}{ Subjective effects } \\
\hline Heart pounding & $6.36(3.43)$ & $8.00(4.99)$ & 0.06 & $16.18(10.59)$ & 1.64 & $54.82(36.44)$ & $53.67 *$ \\
\hline Mellow & $209.55(71.78)$ & $14 \mid .73$ (58.99) & $10.24^{\S}$ & $200.64(64.47)$ & $7.72 *$ & $258.9 \mid(77.78)$ & $30.56 *$ \\
\hline Social & $308.64(66.68)$ & $254.73(53.56)$ & 3.31 & $240.18(45.87)$ & 0.24 & $331.36(64.72)$ & 6.70* \\
\hline Tired & 91.55 (29.90) & $213.18(56.16)$ & $8.88^{\S}$ & $104.00(35.84)$ & $7.15 *$ & I $66.36(47.20)$ & 1.32 \\
\hline Unmotivated & $52.64(35.37)$ & | I 5.00 (44.39) & $7.64^{\S}$ & $56.73(36.04)$ & $6.67 *$ & $54.82(29.86)$ & $7.12 *$ \\
\hline
\end{tabular}

Data are represented as area-under-the-curve values and data from active modafinil conditions are presented for the night shift only.

$\mathrm{df}=1,40$.

$\mathrm{Pbo}=$ placebo; $\mathrm{DAT}=$ divided attention task; $\mathrm{RA}=$ repeated-acquisition task; $\mathrm{RIT}=$ rapid information task

$\S_{p}<0.03$, significant difference between the placebo day and placebo night conditions.

* $p<0.03$, significant difference from the placebo night condition.

prone to work-related accidents and automobile accidents while driving home from night-shift work (Mitler et al, 1988; Leger, 1994).

Most of the effects on cognitive performance following placebo administration persisted across all three nights the participants worked on the night shift. One possible reason for the observed pattern of cognitive decrements is that participants' sleep was disrupted, and this affected next-day performance. The sleep data lend partial support to this perspective, but do not entirely account for the scope of impairments. Nightcap sleep results indicated that participants slept shorter durations and required longer durations to initiate sleep during night-shift work, although the majority of these effects did not reach statistical significance and were most pronounced on the first night. Subjective measures of sleep were consistent with Nightcap data. Subjective ratings of mood during the nighttime work period, by contrast, were more congruent with cognitive performance data. Participants reported being more 'Sleepy' and less motivated throughout all three nights they worked on the night shift. Several other ratings of mood were also disrupted on at least two of the three nights (eg, 'Sedated' and 'Tired'). In an effort to lessen the negative impact of abrupt changes in work-shift schedules, an off day was implemented prior to starting work on a different shift. The current findings suggest that an acclimation period greater than 3 days is necessary when psychostimulant-free individuals are subjected to abrupt work-shift schedule changes.

Modafinil attenuated nearly all night shift-related cognitive/psychomotor impairments in a dose-related manner. For example, both active doses improved performance on the immediate recall and DSSTs, but the effects produced by the 400-mg dose were significantly different from those produced by the 200-mg dose. Other performance effects showed a similar pattern and were in general agreement with the findings of Walsh et al (2004). A divergent finding, however, was noted for modafinil-related effects on DSST performance. In the Walsh et al study modafinil did not improve nighttime DSST performance, whereas in the current investigation it substantially improved performance. An important methodological difference between the two studies might explain this apparent discrepancy. In the earlier study, participants did not receive training on the cognitive tasks before the study began. As a result, DSST performance improved during each of the four nights within each dosing condition, making it more difficult to detect modafinil-associated effects. In contrast, in the present study, participants were trained on the cognitive tasks for 2 days prior to moving into the laboratory. The large amount of training ensured that the tasks were welllearned prior to study participation, so that learning effects would be minimized during the study. This methodological difference could have influenced the results. 
"Alert"

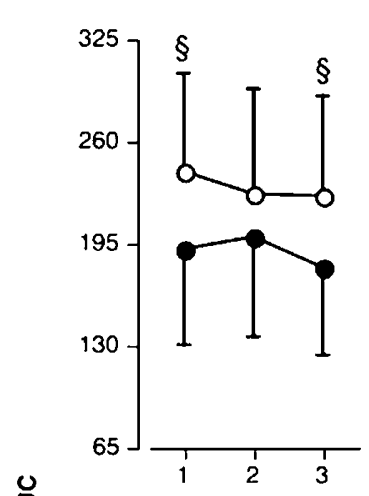

$\stackrel{9}{4}$

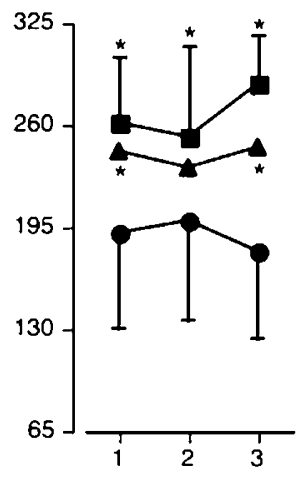

"Can't Concentrate"
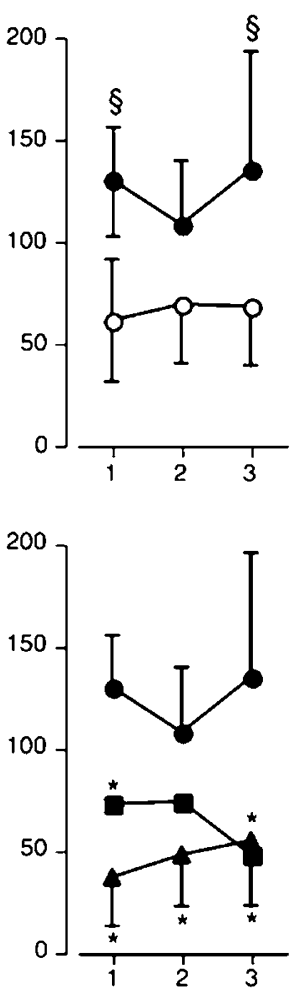

Day within Condition
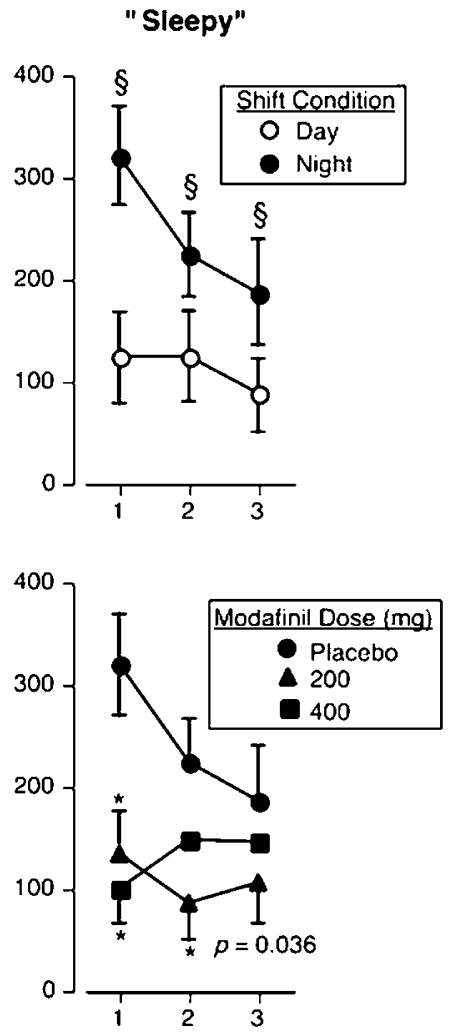

Figure 2 Upper panel: AUC values for visual analog scale ratings of 'Alert,' 'Can't Concentrate,' and 'Sleepy' as a function of shift condition and day within condition. ${ }^{\S}$ Significant difference between the day and night shift conditions for that day following placebo administration $(p<0.03)$. Bottom panel: AUC values for visual analog scale ratings of 'Alert,' 'Can't Concentrate,' and 'Sleepy' as a function of modafinil dose and day of the night-shift condition. *Significant difference between placebo and that dose of modafinil for that day $(p<0.03)$. Error bars represent one SEM. Overlapping error bars were omitted for clarity.

Modafinil also improved performance during day-shift work but to a lesser degree. This observation is in line with other researchers who found that the drug produced limited effects on cognitive performance in healthy, well-rested individuals (eg, Muller et al, 2004; Randall et al, 2004, 2005). By contrast, modafinil produced greater sleep disruptions when participants worked on the day shift than when they worked on the night shift. These effects were unexpected and have not been reported previously with modafinil. The data, however, are partly congruent with previous studies that have evaluated the effects of psychostimulants on sleep (eg, Post et al, 1974; Comer et al, 2001). For example, Comer et al (2001) reported that methamphetamine decreased total sleep time and sleep efficiency on the first day of administration. On the subsequent days, these effects were no longer significant, suggesting the development of tolerance. Perhaps if modafinil was administered on a greater number of consecutive days in the current study, tolerance would have developed to its effects on sleep.

Modafinil improved mood during the night shift, although its effects were less dramatic than those observed on cognitive/psychomotor performance. Both active doses increased subjective reports of alertness and decreased reports of sleepiness on at least two of the three nights. Other ratings of mood were also improved by modafinil treatment, while the drug produced few 'negative' subjective effects. These results are congruent with data from this laboratory demonstrating that methamphetamine attenuated night shift-related mood disruptions (Hart et al, 2003a). It is important to note, however, that modafinil did not increase ratings associated with drug abuse liability. Unlike data from our previous study with methamphetamine, ratings of 'good drug effect' and participants' reported desire to take the drug again were not significantly altered by modafinil. This is consistent with clinical observations (for a review, see Myrick et al, 2004) and human laboratory data (Rush et al, 2002), indicating that the abuse potential of modafinil is low.

The current findings should be interpreted within the context of at least two study limitations. Study participants were not exposed to the natural light/dark cycle, unlike shift workers in their natural ecology. As the natural light/dark cycle is known to influence circadian rhythm alignment, which has been proposed to be an important mediator of shift change-related performance decrements and sleep disruptions (Eastman and Martin, 1999), it is possible that the generality of our findings to many shift workers may be limited. Nevertheless, the current data and those collected in our previous studies (Hart et al, 2003a, b, 2005) demonstrating that performance and mood were disrupted as a function of the night-shift condition argue that the procedures used here provide a useful model to simulate 
Night Cap: Sleep Time

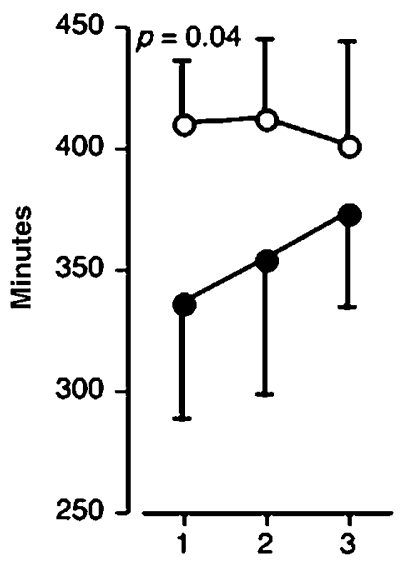

Sleep Questionnaire: Estimated Sleep Time

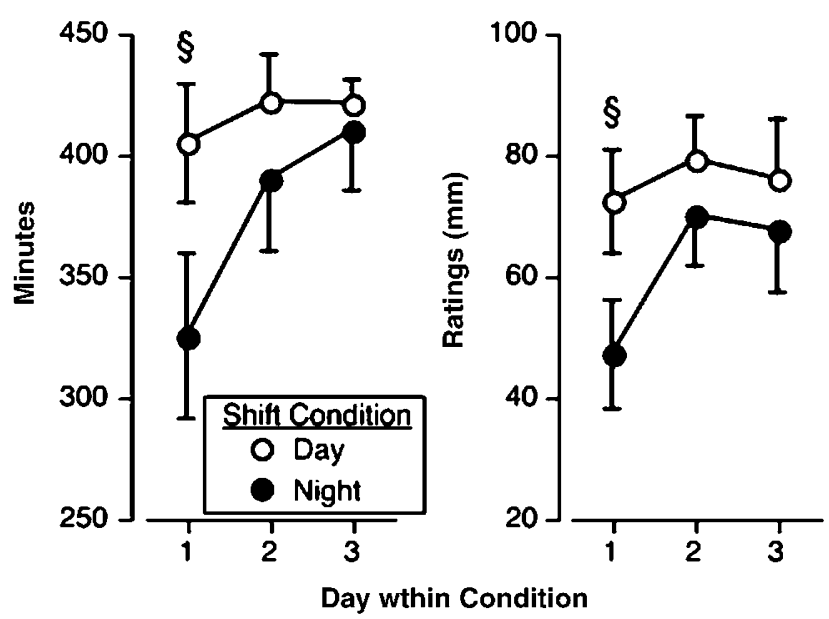

Figure 3 Upper panel: Total sleep time and sleep onset latency from the previous evening as measured by the Nightcap as a function of shift

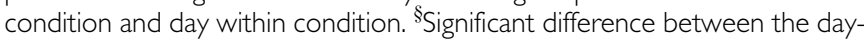
and night-shift conditions for that day following placebo administration $(p<0.03)$. Bottom panel: Selected mean subjective effects from the sleep questionnaire as a function of shift condition and day within condition. \$Significant difference between the day- and night-shift conditions for that day following placebo administration $(p<0.03)$.

work-shift change conditions, and that the effects of medications can be sensitively detected within this model. Another caveat of the current study is that although modafinil is an approved shift work sleep disorder medication, the applicability of these findings to a patient population is unknown because they were obtained in healthy research volunteers.

In conclusion, these data demonstrate that performance, mood, and sleep are disrupted during night-shift work when research participants are subjected to abrupt work schedule changes. The data further show that therapeutic doses of modafinil can decrease night shift-related performance and mood disruptions. These findings may be of particular significance in occupations that require individuals to undergo abrupt changes in work schedules, for example, healthcare workers, military personnel, and police officers.

\section{ACKNOWLEDGEMENTS}

The National Institute on Drug Abuse provided financial support for this research (Grant \#DA-03746). We gratefully acknowledge the assistance of Brooke Roe, Susan Loftus, Diana Paksarian, Jana Colley, Michael Rubin, Liah Barnett, Aleksandra Beselman, and Drs John Mariani and Jeanne Manubay.

\section{REFERENCES}

Ajilore O, Stickgold R, Rittenhouse CD, Hobson JA (1995). Nightcap: laboratory and home-based evaluation of a portable sleep monitor. Psychophysiology 32: 92-98.

Buguet A, Montmayeur A, Pigeau R, Naitoh P (1995). Modafinil, damphetamine and placebo during $64 \mathrm{~h}$ of sustained mental work. II. Effects on two nights of recovery sleep. J Sleep Res 4: 229-241.

Cantero JL, Atienza M, Stickgold R, Hobson JA (2002). Nightcap: a reliable system for determining sleep onset latency. Sleep 25: 238-245.

Comer SD, Hart CL, Ward AS, Haney M, Foltin RW, Fischman MW (2001). Effects of repeated oral methamphetamine administration in humans. Psychopharmacology 155: 397-404.

Crochet S, Sakai K (2003). Dopaminergic modulation of behavioral states in mesopontine tegmentum: a reverse microdialysis study in freely moving cats. Sleep 26: 801-816.

de Saint Hilaire Z, Orosco M, Rouch C, Blanc G, Nicolaidis S (2001). Variations in extracellular monoamines in the prefrontal cortex and medial hypothalamus after modafinil administration: a microdialysis study in rats. Neuroreport 12: 3533-3537.

Dempster FN, Corkill AJ (1999). Individual differences in susceptibility to interference and general cognitive ability. Acta Psychol 101: 395-416.

Duteil J, Rambert FA, Pessonnier J, Hermant JF, Gombert R, Assous E (1990). Central alpha 1-adrenergic stimulation in relation to the behaviour stimulating effect of modafinil; studies with experimental animals. Eur J Pharmacol 180: 49-58.

Eastman CI, Martin SK (1999). How to use light and dark to produce circadian adaptation to night shift work. Ann Med 31: 87-98.

Ferraro L, Antonelli T, O’Connor WT, Tanganelli S, Rambert FA, Fuxe K (1998). The effects of modafinil on striatal, pallidal and nigral GABA and glutamate release in the conscious rat: evidence for a preferential inhibition of striato-pallidal GABA transmission. Neurosci Lett 253: 135-138.

Foltin RW, Haney M, Comer SD, Fischman MW (1996). Effects of fluoxetine on food intake of humans living in a residential laboratory. Appetite 27: 165-181.

Haney M, Ward AS, Comer SD, Hart CL, Foltin RW, Fischman MW (2001). Bupropion SR worsens mood during marijuana withdrawal in humans. Psychopharmacology 155: 171-179.

Hart CL, Haney M, Nasser J, Foltin RW (2005). Combined effects of methamphetamine and zolpidem on behavior of shift workers. Pharm Biochem Behav 81: 559-568.

Hart CL, van Gorp WG, Haney M, Foltin RW, Fischman MW (2001a). Effects of acute smoked marijuana on complex cognitive performance. Neuropsychopharmacology 25: 757-765.

Hart CL, Ward AS, Haney M, Foltin RW (2003a). Zolpidem-related effects on performance and mood during simulated night-shift work. Exp Clin Psychopharmacol 11: 259-268.

Hart CL, Ward AS, Haney M, Foltin RW, Fischman MW (2001b). Methamphetamine self-administration by humans. Psychopharmacology 157: 75-81.

Hart CL, Ward AS, Haney M, Nasser J, Foltin RW (2003b). Methamphetamine attenuates disruptions in performance and mood during simulated night shift work. Psychopharmacology 169: $42-51$. 
Jasinski DR (2000). An evaluation of the abuse potential of modafinil using methylphenidate as a reference. J Psychopharmacol 14: 53-60.

Kelly TH, Foltin RW, Emurian CS, Fischman MW (1993). Performance-based testing for drugs of abuse: dose and time profiles of marijuana, amphetamine, alcohol, and diazepam. J Anal Toxicol 17: 264-272.

Leger D (1994). The cost of sleep-related accidents: a report for the National Commission on Sleep Disorders Research. Sleep 17: 84-93.

Lin JS, Roussel B, Akaoka H, Fort P, Debilly G, Jouvet M (1992). Role of catecholamines in the modafinil and amphetamine induced wakefulness, a comparative pharmacological study in the cat. Brain Res 591: 319-326.

Malenka RC, Nicoll RA (1986). Dopamine decreases the calciumactivated afterhyperpolarization in hippocampal CA1 pyramidal cells. Brain Res 379: 210-215.

McLeod DR, Griffiths RR, Bigelow GE, Yingling J (1982). An automated version of the digit symbol substitution test (DSST). Behav Res Meth Instr 14: 463-466.

Mignot E, Nishino S, Guilleminault C, Dement WC (1994). Modafinil binds to the dopamine uptake carrier site with low affinity. Sleep 17: 436-437.

Miller TP, Taylor JL, Tinklenberg JR (1988). A comparison of assessment techniques measuring the effects of methylphenidate, secobarbital, diazepam, and diphenhydramine in abstinent alcoholics. Neuropsychobiology 19: 90-96.

Mitler MM, Carskadon MA, Czeisler CA, Dement WC, Dinges DF, Graeber RC (1988). Catastrophes, sleep, and public policy: consensus report. Sleep 11: 100-109.

Muehlbach MJ, Walsh JK (1995). The effects of caffeine on simulated night-shift work and subsequent daytime sleep. Sleep 18: 22-29.

Muller U, Steffenhagen N, Regenthal R, Bublak P (2004). Effects of modafinil on working memory processes in humans. Psychopharmacology 177: 161-169.

Myrick H, Malcolm R, Taylor B, LaRowe S (2004). Modafinil: preclinical, clinical, and post-marketing surveillance-a review of abuse liability issues. Ann Clin Psychiatry 16: 101-109.

Ohayon MM, Lemoine P, Arnaud-Briant V, Dreyfus M (2002). Prevalence and consequences of sleep disorders in a shift worker population. J Psychosom Res 53: 577-583.

Pigeau R, Naitoh P, Buguet A, McCann C, Baranski J, Taylor M et al (1995). Modafinil, d-amphetamine and placebo during $64 \mathrm{~h}$ of sustained mental work. I. Effects on mood, fatigue, cognitive performance and body temperature. J Sleep Res 4: 212-228.
Post RM, Gillin JC, Wyatt RJ, Goodwin FK (1974). The effect of orally administered cocaine on sleep of depressed patients. Psychopharmacologia 37: 59-66.

Randall DC, Fleck NL, Shneerson JM, File SE (2004). The cognitiveenhancing properties of modafinil are limited in non-sleepdeprived middle-aged volunteers. Pharmacol Biochem Behav 77: 547-555.

Randall DC, Viswanath A, Bharania P, Elsabagh SM, Hartley DE, Shneerson JM et al (2005). Does modafinil enhance cognitive performance in young volunteers who are not sleep-deprived? J Clin Psychopharmacol 25: 175-179.

Reid K, Dawson D (2001). Comparing performance on a simulated $12 \mathrm{~h}$ shift rotation in young and older subjects. Occup Environ Med 58: 58-62.

Rush CR, Kelly TH, Hays LR, Baker RW, Wooten AF (2002). Acute behavioral and physiological effects of modafinil in drug abusers. Behav Pharmacol 13: 105-115.

Schwartz JR (2005). Modafinil: new indications for wake promotion. Expert Opin Pharmacother 6: 115-129.

Sharkey KM, Fogg LF, Eastman CI (2001). Effects of melatonin administration on daytime sleep after simulated night shift work. J Sleep Res 10: 181-192.

Tallarida RJ, Murray RB (1981). Manual of Pharmacologic Calculations. Springer: Heidelberg, Berlin, New York.

US Congress of Technology Assessment (1991). Biological Rhythms: Implications for the Worker. US Government Printing Office: Washington, DC.

Walsh JK, Muelbach MJ, Humm TM, Dickens QS, Sugerman JL, Schweitzer PK (1990). Effect of caffeine on physiological sleep tendency and ability to sustain wakefulness at night. Psychopharmacology 101: 271-273.

Walsh JK, Randazzo AC, Stone KL, Schweitzer PK (2004). Modafinil improves alertness, vigilance, and executive function during simulated night shifts. Sleep 27: 434-439.

Wesnes K, Warburton DM (1983). Effects of smoking on rapid information processing performance. Neuropsychobiology 9: 223-229.

Wisor JP, Eriksson KS (2005). Dopaminergic-adrenergic interactions in the wake promoting mechanism of modafinil. Neuroscience 132: 1027-1034.

Wisor JP, Nishino S, Sora I, Uhl GH, Mignot E, Edgar DM (2001). Dopaminergic role in stimulant-induced wakefulness. J Neurosci 21: 1787-1794.

Zacks RT, Hasher L (1994). Directed ignoring: inhibitory regulation of working memory. In: Dagenbach D, Carr TJ (eds). Inhibitory Processes in Attention, Memory, and Language. Academic Press: San Diego, CA. pp 241-262. 